www.jmscr.igmpublication.org Impact Factor 5.244

Index Copernicus Value: 83.27 ISSN (e)-2347-176x ISSN (p) 2455-0450 crossrefDOI: _https://dx.doi.org/10.18535/jmscr/v4i11.75

\title{
Randamoized Open Label Comparative Study on Proglumetacindimaleate and Diclofenac Sodium in Osteoarthritis Patients
}

\author{
Authors \\ L. Madhan ${ }^{1}$, A. Moorthi' ${ }^{2}$ K.P. Sampath Kumar ${ }^{3}$ \\ ${ }^{1}$ Department of Pharmacology, Government Coimbatore Medical College, Coimbatore 641014 \\ ${ }^{2}$ Department of Physiology, Government Coimbatore Medical College, Coimbatore 641014 \\ ${ }^{3}$ Department of Pharmacy, Government Coimbatore Medical College, Coimbatore 641014 \\ Corresponding Author
}

L. Madhan

Email: madhanmrth962@gmail.com

\begin{abstract}
The pathogenesis of osteoarthritis $(O A)$ is not clearly understood; inflammatory mediators play an important role in the pathogenesis of OA. Non steroidal anti inflammatory drugs (NSAIDs) the most widely prescribed drugs in OA. The efficacy of two anti-inflammatory drugs proglumetacindimaleate (PRO) and diclofenac sodium (DIC) in controlling the pain response along with diseases was assessed. The study is a progressive study; patients were followed for 4 weeks period to assess the efficacy of the two different treatments. It is an open labeled, randomized comparative study. The drugs used were PRO (300mg, bid) and DIC (50mg, bid) in osteoarthritis patients. Totally 60 patients of both sexes with moderate to severe osteoarthritis were included in this study. Clinical assessment was done based on the following parameters to assess the efficacy of the drugs and as well as the pain perception. X-ray was done to assess the joints and inflammation including swelling of the joints. The laboratory investigation includes routine haematogram, liver function tests, and kidney function test. The pain perception in the patients was measured using pain scale. The results have indicated that in comparison to PRO, DIC had toxicity in liver as observed by increased SGOT

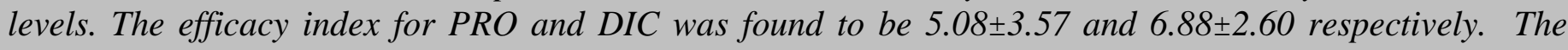
study can be concluded that both PRO and DIC exhibited similar efficacy and controlled the pain perception in OA patients. In any selection of drug of choice for the treatment and analyzing the risk versus benefit PRO has been preferred as NSAIDs due to its better tolerability in GIT complications as well as pro drug property. The precaution to be taken is periodical liver function tests on prolong use of PRO is required.

Keywords- Inflammation, COX inhibitors, Efficacy,Arthritis, analgesics.
\end{abstract}

\section{Introduction}

Osteoarthritis (OA) is one of the common form of arthritis and a leading cause of musculoskeletal pain and disability (Doherty et al., 1998). It is more common in women over the age of 55 years than men (Dieppe 1998). The pathogenesis of OA is not clearly understood; many cases the patients undergo knee replacement surgery and take pain killers. Synovial inflammation is the cause for rapid cartilage degeneration and associated with higher pain response (Scanzello et al., 2008). Hence synovitis has been thought to be an important pathology in OA (Scanzello et al., 2008). 
The disease progression is always associated with the stimulation of Pattern Recognition Receptors (PRRs) by a specific stimulus associated with the disease. In OA deposition of hydroxyapatite (HA) crystals has been recorded in around $60 \%$ of the patients. Positive correlation on disease progression with severity of cartilage degradation (Gibilisco et al., 1985) was observed. HA crystals acting through toll like receptors increased the secretion of IL-1 $\beta$ and IL-18 in murine macrophages(Jin et al., 2011). IL-1 $\beta$ can promote synovitis and cartilage degeneration. Further presence of IL-18 in the cartilage and synovial fluid of OA patients have been linked with disease severity (Jin et al., 2011). Hence controlling the inflammation by IL blockade or IL induced inflammatory response has been found to be an effective therapeutics in OA.

Non steroidal anti inflammatory drugs (NSAIDs) the most widely prescribed drugs in OA. Though NSAIDs couldn't prevent completely the cartilage tissue damage but has been widely used to control inflammatory response mediated by inflammatory mediators. Currently immune modulators, drugs controlling cartilage degradation or slow down and regulating cartilage metabolism, targeting sub arachanoidal bone along with analgesics are the therapeutics (Wang et al., 2015).

In the present study efficacy of two antiinflammatory drugs proglumetacindimaleate and diclofenac sodium in controlling the pain response along with diseases progression were assessed in OA patients. The selected drugs produce analgesics response and controls inflammatory response. The study is a progressive study patient's were followed for 4 weeks period to assess the efficacy of the two different treatments.

\section{MATERIALS AND METHODS \\ Study design}

It is an open labeled, randomized comparative study. The study was carried out to compare the efficacy of proglumetacindimaleate $(300 \mathrm{mg}$, bid) and dicofenac Sodium (50mg, bid) in osteoarthritis patients. The work was done at Department of Pharmacology in collaboration with the Department of Pharmacology, Government Coimbatore Medical College \& Hospital, Coimbatore, Tamilnadu. The approval to conduct the study was obtained from local human ethical committee. The approval detail is CMC/PCOL/02/2013.

\section{Patient's selection}

Totally 60patients of both sexes with moderate to severe osteoarthritis were included in this study. Both old and new cases were recruited for the study and were given a washout period of 1 week followed by a trial period of 4 weeks.

\section{Inclusion Criteria:}

- Male and Female patients above 18 yrs of age.

- Ascertained diagnosis of osteoarthritis with corresponding radiological diagnosis.

\section{Exclusion Criteria:}

- Known hypersensitivity of NSAIDs.

- Serious systemic diseases

- Gastrointestinal bleeding or ulcer

- Female who were pregnant, breast feeding or at risk of pregnancy and not using a medically acceptable form of contraception

- Advanced diabetics patients

- Severe disease of kindly with serum creatinine values more than $1.8 \mathrm{mg} / \mathrm{dl}$ or proteinuria more than ++ , at more than two following tests.

- Liver disease

- Use of any investigational drug within 30 days prior to the screening visit

\section{Investigational parameters}

Clinical assessment was done based on the following parameters to assess the efficacy of the drugs and as well as the pain perception. X-ray was done to assess the joints and inflammation including swelling of the joints

Laboratory investigations- routine haemogram such as $\mathrm{Hb}$, total counts, differential counts, ESR and PCV including platelet counts were done in both the groups. The other biochemical 
parameters measured includes liver function test (SGOT, SGPT, total bilirubin, $\mathrm{S}$ protein and $\mathrm{S}$ albumin), blood sugar, blood urea, S. creatinine and stools for occult blood.

\section{Clinical parameters}
A. Parameters
1) No. of painful joints
2) No. of swollen joints
3) Duration of morning stiffness in minutes.
4) Time to walk 50 feet in seconds.
B. Signs and Symptoms
1) Pain
2) Pain on movement
3) Tenderness
4) Swelling
5) Erythema
6) Ability to perform physical activity.

\section{Pain scale and measurements}

Pain and pain on movement was evaluated by four point scale ( 0 to 3 ) $-0=$ absent; $1=$ no interference with daily activity; $2=$ some interference with daily activity; $3=$ incapacitations

Tenderness was evaluated by four-point scale $(0$ to 3$)-0=$ no pain; $1=$ palpable and visible; $3=$ distortion of joint centers

Swelling was evaluated by four-point scale ( 0 to 3) $-0=$ none; $1=$ palpable; $2=$ palpable and visible; $3=$ distortion of joint centers]

Physical activity was evaluated by four-point scale (0 to 3$)-0=$ no discomfort; $1=$ some discomfort; $2=$ discomfort and difficulty; $3=$ not possible]

\section{Statistical analysis}

Data were expressed as mean \pm SD. Statistical significance between the groups in clinical and biochemicals were analyzed by student ' $t$ ' testspaired using Graph Pad Prism, 4.03 (San Diego, US). The efficacy between the groups was compared by unpaired student ' $t$ ' tests. Statistical significance at $\mathrm{P}<0.05$ was considered as statistical significance.

\section{Results \\ Demographic details}

The mean age and weight of PRO group was 53.46 years (range $34-70$ years) and $61.37 \mathrm{~kg}$ respectively. The DIC group had mean age of 54.72 years (range $30-73$ years) and $58.96 \mathrm{~kg}$. The systolic / diastolic BP and height has no significant difference between the groups. The duration of OA illness as per history was found to be $($ mean \pm SD) $33.0 \pm 22.7$ and 34.0 \pm 29.9 months for PRO and DIC group respectively. In PRO group out of 30 patients only 25 completed the study and 5 patients did not turn up after the first week of drug therapy. In DIC group out of 30 patients, 25 completed the study. Three patients were not turned up for the follow up study and 2 patients discontinued due to severe gastritis in the $2^{\text {nd }}$ week of the study.

\section{Haemogram}

Patients received PRO; measurement of routine haemogram denotes no significant difference from base line versus four weeks treatment. In comparison to base line value at $4^{\text {th }}$ week significant elevation in SGOT (25.8 \pm 4.3 against $27.9 \pm 5.0 ; \mathrm{p}<0.05), \quad$ SGPT $(23.24 \pm 8.36$ against $26.88 \pm 9.0 ; \mathrm{p}<0.05)$ and BUN $(24.7 \pm 3.2$ against $23.5 \pm 3.1 ; \quad \mathrm{p}<0.05)$ was observed. All other biochemicall parameters remain unaltered with PRO treatment.

Patients received DIC; haemogram parameters remain unaltered except ESR. One hour of ESR value was found to be increased at $4^{\text {th }}$ week $(11.42 \pm 7.3$ against $16.7 \pm 15.9 ; \quad \mathrm{p}<0.05)$. The clinical biochemical parameters and liver function tests exhibited normal values upon 4 weeks DIC treatment in OA patients.

\section{Pain evaluation}

The efficacy of the drugs treatment in OA patients were assessed with parameters such as number of painful joints, swollen joints, morning stiffness and time taken to cover the 50 feet distance. The results denotes both PRO and DIC treatments improved the symptoms at $4^{\text {th }}$ week of the study as indicated by the subjective scores like decreased number of painful joints and swollen 


\section{JMSCR Vol ||04||Issue ||11||Page 13961-13966||November}

joints, morning stiffness and less time taken to walk 50 feet distance in comparison to base line value. Both the drugs have shown gradual decrease in the pain perception and were effective only at $4^{\text {th }}$ week. No significant difference between the drugs in different weeks of treatment was observed.

Signs and symptoms with respective to PRO and DIC treatment was evaluated scoring pain perception, tenderness, swelling and physical activity initiation after the command. A gradual decrease in the pain and pain on movement was observed as evidenced by decreased pain scores with treatment progression. Tenderness and swelling scores indicate reduction at $2^{\text {nd }}$ week of the treatment followed by gradual and slow recovery on these two parameters with $\mathrm{PRO}$ and DIC treatment. The time taken for ability to perform physical activity upon command was found to be decreased indicates that muscle stiffness and pain in the joints and movements are reduced. All the parameters studied have shown significant recovery of OA condition at $4^{\text {th }}$ week. Both the treatments have produced similar effects.

\section{Efficacy assessment}

The efficacy index was calculated at $4^{\text {th }}$ week of the study based on clinical global impression scale. Accordingly therapeutic efficacy was classified as marked, moderate and minimal or NIL with or without side effects. End of the study the efficacy index for PRO and DIC was found to be $5.08 \pm 3.57$ and $6.88 \pm 2.60$ respectively. In comparison to base line the efficacy index value at the end of the study has been improved. In this parameter also both the drugs have behaved similarly and no significant difference in efficacy measured as pain response was observed.

Table 1- Effect of proglumetacindimaleateand diclofenac sodium on efficacy parameters in OA patients Data are expressed a Mean \pm SD. Superscript *denotes statistical significance $\mathrm{P}<0.05$ versus base line observation.

\begin{tabular}{|l|l|l|l|l|l|l|l|l|}
\hline Duration & \multicolumn{4}{l|}{ Proglumetacindimaleate } & \multicolumn{3}{l|}{ Diclofenac sodium } \\
\hline & 1 & 2 & 3 & 4 & 1 & 2 & 3 & 4 \\
\hline Base & $1.96 \pm$ & $0.88 \pm$ & $1.82 \pm$ & $58.68 \pm$ & $2.08 \pm$ & $1.16 \pm$ & $1.72 \pm$ & $64.92 \pm$ \\
& 0.78 & 0.93 & 2.29 & 24.87 & 0.70 & 0.90 & 2.03 & 27.41 \\
\hline $1^{\text {st }}$ week & $1.76 \pm$ & $0.56 \pm$ & $1.24 \pm$ & $52.32 \pm$ & $2.04 \pm$ & $0.80 \pm$ & $1.40 \pm$ & $60.48 \pm$ \\
& 0.44 & 0.82 & 2.07 & 20.62 & 0.68 & 0.91 & 1.78 & 26.31 \\
\hline $2^{\text {nd }}$ week & $1.56 \pm$ & $0.52 \pm$ & $0.92 \pm$ & $46.76 \pm$ & $1.92 \pm$ & $0.56 \pm$ & $1.20 \pm$ & $56.44 \pm$ \\
& 0.44 & 0.77 & 1.50 & 14.52 & 0.40 & 0.77 & 1.53 & 24.44 \\
\hline $3^{\text {rd }}$ week & $1.40 \pm$ & $0.32 \pm$ & $0.56 \pm$ & $46.44 \pm$ & $1.76 \pm$ & $0.48 \pm$ & $1.08 \pm$ & $54.68 \pm$ \\
& 0.64 & 0.69 & 1.04 & 14.65 & 0.66 & 0.71 & 1.47 & 22.29 \\
\hline $4^{\text {th }}$ week & $1.08 \pm$ & $1.12 \pm$ & $0.48 \pm$ & $46.36 \pm$ & $1.60 \pm$ & $0.36 \pm$ & $1.08 \pm$ & $53.40 \pm$ \\
& $0.81^{*}$ & $0.44^{*}$ & $0.96^{*}$ & $14.72^{*}$ & $0.58^{*}$ & $0.64^{*}$ & $1.47^{*}$ & $21.14^{*}$ \\
\hline
\end{tabular}

Table 2- Effect of proglumetacin dimaleate and diclofenac sodium on signs and symptoms parameters in OA patients Data are expressed a Mean \pm SD. Superscript *denotes statistical significance $\mathrm{P}<0.05$ versus base line observation.

\begin{tabular}{|l|l|l|l|l|l|l|l|l|}
\hline Duration & \multicolumn{2}{l|}{ Proglumetacindimaleate } & \multicolumn{4}{l|}{ Diclofenac sodium } \\
\hline & 1 & 2 & 3 & 4 & 1 & 2 & 3 \\
\hline Base & $2.00 \pm$ & $1.44 \pm$ & $0.68 \pm$ & $1.92 \pm$ & $2.04 \pm$ & $1.44 \pm$ & $1.04 \pm$ & $1.96 \pm$ \\
& 0.00 & 0.8 & 0.80 & 0.28 & 0.20 & 0.82 & 0.84 & 0.20 \\
\hline $1^{\text {st }}$ week & $1.68 \pm$ & $1.00 \pm$ & $0.44 \pm$ & $1.80 \pm$ & $1.96 \pm$ & $1.20 \pm$ & $0.60 \pm$ & $1.88 \pm$ \\
& 0.48 & 0.82 & 0.71 & 0.50 & 0.20 & 0.87 & 0.71 & 0.33 \\
\hline $2^{\text {nd }}$ week & $1.48 \pm$ & $0.68 \pm$ & $0.36 \pm$ & $1.44 \pm$ & $1.76 \pm$ & $0.96 \pm$ & $0.44 \pm$ & $1.76 \pm$ \\
& 0.58 & 0.63 & 0.57 & 0.88 & 0.43 & 0.84 & 0.58 & 0.44 \\
\hline $3^{\text {rd }}$ week & $1.24 \pm$ & $0.52 \pm$ & $0.36 \pm$ & $1.20 \pm$ & $1.36 \pm$ & $0.72 \pm$ & $0.28 \pm$ & $1.48 \pm$ \\
& 0.60 & 0.65 & 0.76 & 0.64 & 0.49 & 0.79 & 0.54 & 0.51 \\
\hline $4^{\text {th }}$ week & $0.96 \pm$ & $0.56 \pm$ & $0.20 \pm$ & $1.08 \pm$ & $1.28 \pm$ & $0.72 \pm$ & $0.16 \pm$ \\
& $0.79^{*}$ & $0.71^{*}$ & $0.71^{*}$ & $0.76^{*}$ & $0.54^{*}$ & $0.79^{*}$ & $0.37^{*}$ & $1.32 \pm$ \\
\end{tabular}


Figure 1

\section{Global assessment}

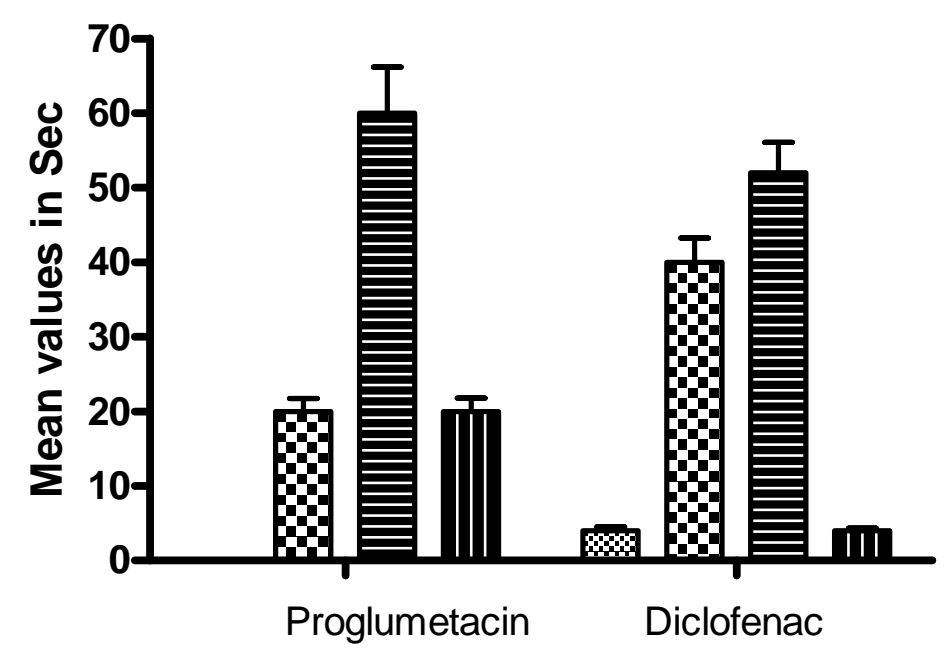

Pon Poor

Deair

= Good

IIII Excellent

Figure 2 Severity of illness

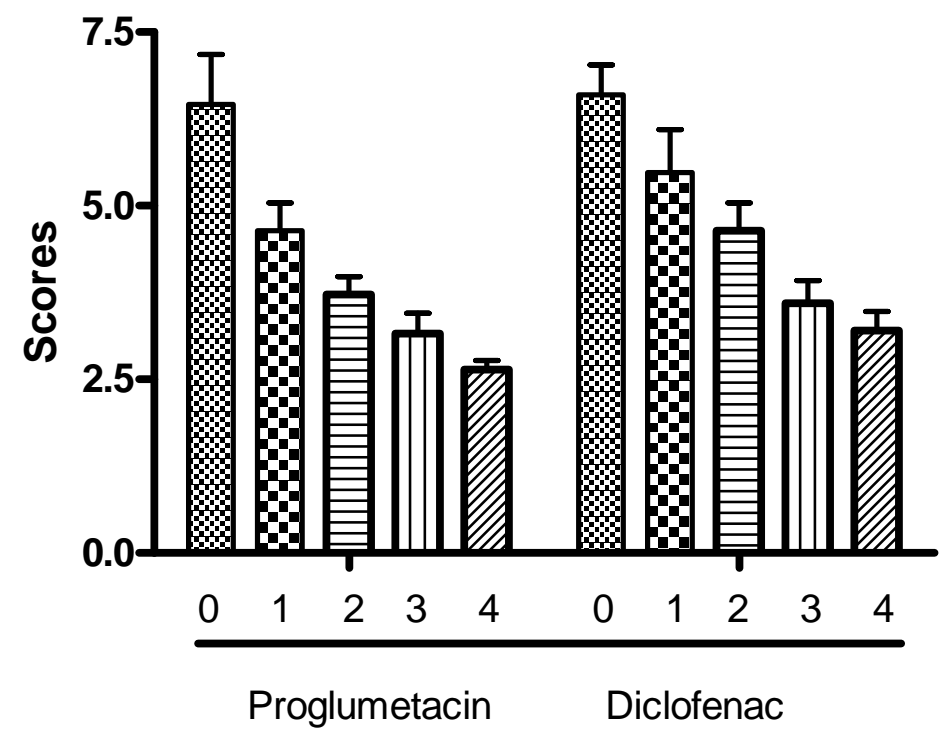

\section{Discussion}

In OA patients the most widely used therapy includes NSAIDs to overcome pain symptoms. In the present study both PRO and DIC behaved similarly and reduced the pain perception indicates equivocal effect. In earlier clinical comparative studies have indicated that PRO (150 $\mathrm{mg}$, bid) with naproxen (250mg, bid) have shown no significant difference in pain perception at rest, during movements and under pressure except during night hours(Sileghem et al., 1991). In RA patients PRO (150mg, bid) and indomethacin (50mg, tid) did not show significant difference in the management of RA (Boom and Franchimont 1994). These observations support our present study. However, one clinical study in acute ankle sprain patients has shown better effect of over DIC, however PRO exhibited more GIT complaints (Simon et al., 1993).

The advantage of PRO is as pro drug. Being it is a pro drug the expectation is reduced GIT complaints. In the present study no major GIT 
complaints have been reported by the OA patients. However study has shown GI complaints with PRO (Simon et al., 1993). However, PRO patients exhibited increased SGOT, SGPT and BUN levels. The elevated levels are within the normal whereas in DIC patients throughout shown increased liver function tests and three were withdrawn from the study due to gastric irritation. Blood parameters and other biochemical parameters did not differ between the groups indicate these drugs are not influencing kidney and protein levels. PRO and DIC produced antiinflammatory response through COX enzymes. Hence the efficacy did not differ. To further elucidate the mechanism of action of these drugs measurement of cytokines will be useful.

The study can be concluded that both PRO and DIC exhibited similar efficacy and controlled the pain perception in OA patients. In any selection of drug of choice for the treatment and analyzing the risk versus benefit PRO has been preferred as NSAIDs due to its better tolerability in GIT complications as well as prodrug property. The precaution to be taken is periodical liver function tests on prolong use of PRO is required.

\section{References}

1. Dieppe P. Osteoarthritis and related disorders. Disorders of bone, cartilage and connective tissue. John H. Klippel, Paul A. Diepee. Rheumatology, Vol.2, $2^{\text {nd }}$ ed. 1998, London, P. 8/1.1 -8/1.2.

2. Doherty M, Jones A, Cawston T.E. Osteoarthritis. Peter J.Mudison et al. Oxford Text book of Rheumatology. $2^{\text {nd }}$ ed. 1998, Oxford University press. New York. P. 151, 1540.

3. Gibilisco PA, Schumacher HR, Jr, Hollander JL, Soper KA. Synovial fluid crystals in osteoarthritis. Arthritis Rheum. 1985;28:511-515.

doi: 10.1002/art.1780280507.

4. Jin C, Frayssinet P, Pelker R, Cwirka D, $\mathrm{Hu}$ B, Vignery A, et al. NLRP3 inflammasome plays a critical role in the pathogenesis of hydroxyapatite-associated arthropathy. ProcNatlAcadSci U S A. 2011;108:14867-14872.

doi: 10.1073/pnas.1111101108.

5. Scanzello CR, Plaas A, Crow MK. Innate immune system activation in osteoarthritis: is osteoarthritis a chronic wound? Curr Opin Rheumatol. 2008;20:565-572. doi: 10.1097/BOR.0b013e32830aba34.

6. Sileghem. A, Verstreten. A, Dequeker J. Double blind randomized, parallel group study of the efficacy and safety of proglumetacin and naproxen in periarthritis of shoulder or elbow. Current Therapeutic Research 1991:50(1):93-94.

7. Wang $\mathrm{K}, \mathrm{Xu} \mathrm{J}$, Hunter DJ, Ding $\mathrm{C}$. Investigational drugs for the treatment of osteoarthritis. Expert OpinInvestig Drugs. 2015;24(12):1539-56.

doi:10.1517/13543784.2015.1091880.

Epub 2015 Oct 1. Review. PubMed PMID: 26429673.

8. Simon J.Brodsky. A, Famey. JJ. P, Randomized double blind study of the efficacy and tolerability of proglumetacin versus diclofenac in sports trauma. Current therapeutic Research 1993:53 (6):630.

9. Boom TA, Franchimont P, Proglumetacin versus Indomethacin in Rheumatoid arthritis. A double blind multicentre study. Advances in Therapy 1994:11(5):228. 